\title{
Dilated tonic pupils with tabes dorsalis in neurosyphilis as first manifestation of HIVIAIDS: a video report
}

\author{
Antonio Jose Reyes, ${ }^{1,2}$ Kanterpersad Ramcharan, ${ }^{1}$ Samuel Aboh, ${ }^{2}$ \\ Stanley Lawrence Giddings ${ }^{2,3}$
}

$>$ Additional material is published online only. To view please visit the journal online (http://dx.doi.org/10.1136/ bcr-2017-223727).

${ }^{1}$ Neurology Unit, Department of Medicine, San Fernando Teaching Hospital, San Fernando, Trinidad and Tobago 'Infectious Disease Unit, Department of Medicine, San Fernando Teaching Hospital, San Fernando, Trinidad and Tobago ${ }^{3}$ Department of Medicine, University of the West Indies, St Augustine, Trinidad and Tobago

Correspondence to Dr Kanterpersad Ramcharan, kramcharan79@yahoo.com

Accepted 22 December 2017

\section{DESCRIPTION}

A previously healthy 30-year-old bisexual African man was admitted with a 3-month history of weight loss, paroxysmal shooting pains and paraesthesia of both lower limbs and difficulty in walking. $\mathrm{He}$ denied progression of visual symptoms such as blurred vision with reading and near work, photophobia, anisocoria or visual loss. The Mini-Mental State Examination was normal $(30 / 30)$. He was alert, oriented to time, person and place with a Glasgow Coma Scale of $15 / 15$. His body mass index was $17 \mathrm{~kg} / \mathrm{m}^{2}$. There was generalised wasting syndrome, cervical lymphadenopathy and diffuse hair loss of the scalp and eyebrows. There was no urinary incontinence.

Visual acuity without correction (20/20), visual fields, intraocular pressures $(14 \mathrm{~mm} \mathrm{Hg})$ and fundoscopy were normal in both eyes. Ocular motility was full bilaterally with no nystagmus. The pupils, however, were $6.0 \mathrm{~mm}$ in diameter on room light bilaterally, unreactive to light or accommodation (Video 1, segment 1). Slit lamp examination of the anterior segment was within normal limits in both eyes. Administration of eye drops with $0.125 \%$ pilocarpine produced bilateral pupillary constriction demonstrating cholinergic sensitivity (Video 1, segment 2). Gait was ataxic. Romberg's sign was positive. Muscle power in all limbs were grade 5/5 (Medical Research Council Scale) with normal tone. Babinski sign was negative with hyporeflexia

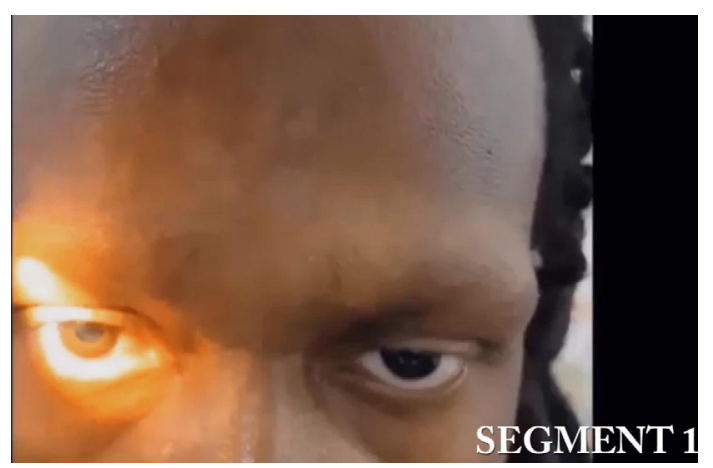

Video 1 Segment 1 showing the pupils at the room light measuring approximately $6.0 \mathrm{~mm}$ in diameter bilaterally, clinically unreactive to light or accommodation, with no constriction even with prolonged near effort. Segment 2 showing bilateral pupillary constriction after eye drops with $0.125 \%$ pilocarpine. The results indicated bilateral tonic pupils. in patellar and Achilles tendons bilaterally. Posterior column sensation for light touch, joint position and vibration sensation were impaired below the anterior superior iliac spine. The rest of the examination was normal.

Serology for HIV ELISA and Venereal Disease Research Laboratory (VDRL) testing (1:64 dilutions) and fluorescent Treponema pallidum antibody absorption (FTA-ABS) test $(4+)$ were positive confirming HIV/AIDS with syphilis coinfection. VDRL test (1:32 dilution) and FTA-ABS test $(3+)$ were positive in cerebrospinal fluid (CSF). The HIV viral load was 356398 RNA copies $/ \mathrm{mL}$, and the CD4 +Tcell count was $110 \mathrm{cells} / \mu \mathrm{L}$ (reference values $410-1590$ ). The international HIV Dementia Scale was normal (12/12). Extensive medical investigations results are shown in table 1. CSF analysis revealed clear, colourless fluid with normal opening pressure $(15 \mathrm{~cm}$ of $\mathrm{H}_{2} \mathrm{O}$ ), moderate pleocytosis with $0.042 \times 10^{9} \mathrm{~L}$ white cell count, $81 \%$ lymphocytes, $9 \%$ polymorphonuclear cells, $10 \%$ monocytes), slightly elevated total protein $(85 \mathrm{mg} / \mathrm{dL})$, normal glucose $60 \mathrm{mg} / \mathrm{dL}$, no bacterial growth and negative India ink for Cryptococcus neoformans. Craniospinal MRI was normal.

Diagnosed as neurosyphilis with HIV/AIDS, he was treated with highly active antiretroviral therapy (HAART), high dosage of intravenous crystalline penicillin, HIV opportunistic infections prophylaxis, pregabalin for neuropathic leg pains and physiotherapy and nutritional support (table 2). Ophthalmological abnormalities resolved after 7 days but lymphadenopathy and wasting syndrome also resolved over 3 months. However, after 8 months of follow-up and being on HAART, tabes dorsalis remained. Repeated HIV viral load at 8 months showed no RNA copies $/ \mathrm{mL}$, and CD4 + Tcell count increased to 375 cells $/ \mu \mathrm{L}$. The serum VDRL level fell, and the CSF VDRL became negative. The percentage of neurosyphilis patients having pupillary abnormalities ranges from $45 \%$ to $53 \%$, although during the course of tabes dorsalis the percentage may be $90 \% \cdot{ }^{1-3} \mathrm{~A}$ unilateral dilated pupil may be seen in early neurosyphilis, prompting timely diagnosis and treatment, but bilateral tonic pupils can occur later.

Contributors AJR, KR, SA and SLG each made substantial contributions to the conception and design of the work, the acquisition, analysis and interpretation of data; they were responsible for drafting the work and revised it critically for important intellectual content; provided final approval of the version published; agreed to be accountable for all aspects of the work; agreed to ensure that questions related to the accuracy 


\begin{tabular}{|c|c|c|}
\hline Blood test & Result & Reference range \\
\hline WCC & $11.6 \times 10^{9} / \mathrm{L}$ & $4.5-11.0 \times 10^{9} / \mathrm{L}$ \\
\hline HGB & $14.2 \mathrm{~g} / \mathrm{dL}$ & $14.0-17.5 \mathrm{~g} / \mathrm{dL}$ \\
\hline MCV & $83.2 \mathrm{fL} / \mathrm{red}$ cell & $80-96 \mathrm{fL} / \mathrm{red}$ cell \\
\hline Platelet count & $249 \times 10^{3} / \mu \mathrm{L}$ & $156-373 \times 10^{3} / \mu \mathrm{L}$ \\
\hline Serum potassium & $4.1 \mathrm{mmol} / \mathrm{L}$ & $3.5-5.1 \mathrm{mmol} / \mathrm{L}$ \\
\hline Serum sodium & $142 \mathrm{mmol} / \mathrm{L}$ & $135-145 \mathrm{mmol} / \mathrm{L}$ \\
\hline Serum creatinine & $0.7 \mathrm{mg} / \mathrm{dL}$ & $0.5-1.2 \mathrm{mg} / \mathrm{dL}$ \\
\hline BUN & $11 \mathrm{mg} / \mathrm{dL}$ & $3-20 \mathrm{mg} / \mathrm{dL}$ \\
\hline Uric acid & $3.1 \mathrm{mg} / \mathrm{dL}$ & $2.5-8 \mathrm{mg} / \mathrm{dL}$ \\
\hline Alanine aminotransferase & $42 \mathrm{IU} / \mathrm{L}$ & $20-60 \mathrm{IU} / \mathrm{L}$ \\
\hline Aspartase aminotransferase & $32 \mathrm{IU} / \mathrm{L}$ & $5-40 I U / L$ \\
\hline Gamma glutamyl transpeptidase & $30 \mathrm{U} / \mathrm{L}$ & 8-61 IU/L \\
\hline Lactate dehydrogenase & $650 \mathrm{IU} / \mathrm{L}$ & $105-333$ IU/L \\
\hline Alkaline phosphatase & $262 \mathrm{U} / \mathrm{L}$ & 40-129 IU/L \\
\hline Total bilirubin & $1.2 \mathrm{mg} / \mathrm{dL}$ & $0.2-1.3 \mathrm{mg} / \mathrm{dL}$ \\
\hline Direct bilirubin & $0.41 \mathrm{mg} / \mathrm{dL}$ & $0.0-0.4 \mathrm{mg} / \mathrm{dL}$ \\
\hline Albumin & $3.5 \mathrm{~g} / \mathrm{dL}$ & $3.5-5.5 \mathrm{~g} / \mathrm{dL}$ \\
\hline Albumin-corrected calcium & $10.94 \mathrm{mg} / \mathrm{dL}$ & $9.6-11.2 \mathrm{mg} / \mathrm{dL}$ \\
\hline CRP & $51.1 \mathrm{mg} / \mathrm{dL}$ & $0.0-1.0 \mathrm{mg} / \mathrm{dL}$ \\
\hline Rheumatoid factor & $36 \mathrm{IU} / \mathrm{mL}$ & Negative: less than $40 \mathrm{IU} / \mathrm{mL}$ \\
\hline Fasting glycaemia & $67 \mathrm{mg} / \mathrm{dL}$ & $60-120 \mathrm{mg} / \mathrm{dL}$ \\
\hline Vitamin $B_{12}$ level & $669 \mathrm{pg} / \mathrm{mL}$ & $200-900 \mathrm{pg} / \mathrm{mL}$ \\
\hline Folic acid level & $6.7 \mathrm{ng} / \mathrm{mL}$ & $2.7-17.0 \mathrm{ng} / \mathrm{mL}$ \\
\hline Erythrocyte sedimentation rate & $8 \mathrm{~mm} /$ hour & Normal: less than $15 \mathrm{~mm} / \mathrm{hour}$ \\
\hline International normalised ratio & 1.00 & $0.5-1.1$ \\
\hline VDRL test & Reactive & Non-reactive or reactive \\
\hline FTA-ABS & Positive (4+) & Positive or negative \\
\hline HIV ELISA & Positive & Positive or negative \\
\hline HIV viral load on admission & 356398 RNA copies/mL & - \\
\hline $\begin{array}{l}\text { HIV viral load } 8 \text { months from } \\
\text { discharge }\end{array}$ & Not detected & - \\
\hline CD4+T cell count on admission & 110 cells/ $\mu \mathrm{L}$ & $410-1590$ cells $/ \mu \mathrm{L}$ \\
\hline $\begin{array}{l}\text { CD4+T cell count } 8 \text { months from } \\
\text { discharge }\end{array}$ & 375 cells/ $/ \mathrm{L}$ & $410-1590$ cells $/ \mu \mathrm{L}$ \\
\hline Antistreptolysin 0 titre & $73 \mathrm{IU} / \mathrm{mL}$ & $0-200 \mathrm{IU} / \mathrm{mL}$ \\
\hline Toxoplasma gondii IgG antibodies & 0.19 & Negative: less than 0.55 \\
\hline T. gondii IgM antibodies & 0.35 & Negative: less than 0.55 \\
\hline Herpes virus $1 \mathrm{lgG}$ antibodies & Less than 0.9 & Index negative: less than 0.9 \\
\hline Herpes virus $1 \lg M$ antibodies & Less than 0.9 & Index negative: less than 0.9 \\
\hline Herpes virus $2 \mathrm{lgG}$ antibodies & Less than 0.9 & Index negative: less than 0.9 \\
\hline Herpes virus $2 \lg M$ antibodies & Less than 0.9 & Index negative: less than 0.9 \\
\hline CMV IgG antibodies & $0.960 \mathrm{UA} / \mathrm{mL}$ & Negative: less than $1.5 \mathrm{UA} / \mathrm{mL}$ \\
\hline CMV IgM antibodies & $0.716 \mathrm{UA} / \mathrm{mL}$ & Negative: less than $1.1 \mathrm{UA} / \mathrm{mL}$ \\
\hline EBV IgG antibodies, & 19 & Positive: greater than 22 \\
\hline EBV IgM antibodies, & 0.5 & Negative: less than 0.8 \\
\hline Hepatitis BS AG & Negative & Positive or negative \\
\hline Hepatitis C IgG antibodies & Negative & Positive or negative \\
\hline Hepatitis C IgM antibodies & Negative & Positive or negative \\
\hline Antidouble-stranded DNA & Negative & Positive or negative \\
\hline Antinuclear antibody & Negative & Positive or negative \\
\hline Antinuclear factor & Negative & Positive or negative \\
\hline $\begin{array}{l}\text { Perinuclear antineutrophil } \\
\text { cytoplasmic antibodies }\end{array}$ & $5.41 \mathrm{U} / \mathrm{mL}$ & Negative: less than $10.0 \mathrm{U} / \mathrm{mL}$ \\
\hline $\begin{array}{l}\text { Cytoplasmic antineutrophil } \\
\text { cytoplasmic antibodies }\end{array}$ & $6.23 \mathrm{U} / \mathrm{mL}$ & Negative: less than $10.0 \mathrm{U} / \mathrm{mL}$ \\
\hline Prostate specific antigen test & $0.1 \mathrm{ng} / \mathrm{mL}$ & Negative: less than $4.0 \mathrm{ng} / \mathrm{mL}$ \\
\hline PCR for viral infections & Tests not obtained & Negative or positive \\
\hline HTLV 1 and 2 antibodies & Negative & Positive or negative \\
\hline $\begin{array}{l}\text { Urine test for cocaine, } \\
\text { amphetamines, opiates, } \\
\text { phencyclidine and } \\
\text { tetrahydrocannabinol }\end{array}$ & Negative & Positive or negative \\
\hline
\end{tabular}

Continued

\begin{tabular}{|c|c|c|}
\hline Blood test & Result & Reference range \\
\hline Other investigations & Result & Reference range \\
\hline Mantoux test & Normal & Normal or abnormal \\
\hline ECG & Normal & Normal or abnormal \\
\hline Chest X-ray & Normal & Normal or abnormal \\
\hline Echocardiogram & Normal EF 75\% & Normal or abnormal \\
\hline $\begin{array}{l}\text { Doppler ultrasound of both lower } \\
\text { limbs }\end{array}$ & Normal & Normal or abnormal \\
\hline MRI scan of spine on admission & Normal & Normal or abnormal \\
\hline $\begin{array}{l}\text { Repeated MRI scan of spine } \\
8 \text { months from discharged }\end{array}$ & Normal & Normal or abnormal \\
\hline $\begin{array}{l}\text { Lumbar puncture for CSF analysis } \\
\text { performed on day } 3 \text { from admission }\end{array}$ & Abnormal & Normal or abnormal \\
\hline Fluid characteristic & Clear and colourless & Normal or abnormal \\
\hline Opening CSF pressure & $15 \mathrm{~cm}$ of $\mathrm{H}_{2} \mathrm{O}$ & $6-25 \mathrm{~cm}$ of water \\
\hline Cell count & $\begin{array}{l}42 \mathrm{WCC} / \mathrm{mm}^{3} \text { ( } 81 \% \\
\text { lymphocytes, } 9 \% \\
\text { polymorphonuclear } \\
\text { cells, } 10 \% \text { monocytes) }\end{array}$ & Normal: $0-5$ cells $/ \mathrm{mm}^{3}$ \\
\hline Protein & $85 \mathrm{mg} / \mathrm{dL}$ & $5-40 \mathrm{mg} / \mathrm{dL}$ \\
\hline Glucose & $60 \mathrm{mg} / \mathrm{dL}$ & $50-80 \mathrm{mg} / \mathrm{dL}$ \\
\hline Culture & No bacterial growth & $\begin{array}{l}\text { No bacterial growth or bacterial } \\
\text { growth }\end{array}$ \\
\hline Cytology & $\begin{array}{l}\text { Negative for neoplastic } \\
\text { cells }\end{array}$ & $\begin{array}{l}\text { Negative or positive for } \\
\text { neoplastic cells }\end{array}$ \\
\hline $\begin{array}{l}\text { India ink for Cryptococcus } \\
\text { neoformans }\end{array}$ & Negative & Positive of negative \\
\hline VDRL test in CSF fluid & Reactive & Non-reactive or reactive \\
\hline FTA-ABS test in CSF fluid & Positive (3+) & Positive or negative \\
\hline
\end{tabular}

CRP, C reactive protein; CSF, cerebrospinal fluid; EBV, Epstein-Barr virus; FTA-ABS, fluorescent Treponema pallidum antibody absorption; $\mathrm{HGB}$, haemoglobin; $\mathrm{MCV}$, mean corpuscular volume; VDRL, Venereal Disease Research Laboratory; WCC, white cell count. BS AG, B surface antigen; BUN, blood urea nitrogen; EF, ejection fraction; HTLV, human T-cell lymphotropic virus.

Table 2 Medical treatment

\begin{tabular}{|c|c|c|}
\hline Intravenous therapy & Dosage & Period of treatment \\
\hline $\begin{array}{l}\text { Aqueous crystalline penicillin } \\
\mathrm{G}\end{array}$ & 4 million units every 4 hours & 14 days \\
\hline $\begin{array}{l}\text { Normal saline intravenous } \\
\text { solution }\end{array}$ & $1 \mathrm{~L}$ daily & 3 days \\
\hline Subcutaneous drug & Dosage & Period of treatment \\
\hline Enoxaparin & 40 units daily & 2 weeks \\
\hline Oral drug & Dosage & Period of treatment \\
\hline Efavirenz & $600 \mathrm{mg}$ daily & 8 months \\
\hline $\begin{array}{l}\text { Tenofovir disoproxil- } \\
\text { emtricitabine }\end{array}$ & $\begin{array}{l}245 / 200 \mathrm{mg} \text { per tablet, } 1 \\
\text { tablet daily }\end{array}$ & 8 months \\
\hline Paracetamol & $1 \mathrm{~g}$ every 6 hours & 3 weeks \\
\hline Pantoprazole & $40 \mathrm{mg}$ once daily & 1 week \\
\hline $\begin{array}{l}\text { Trimethoprim- } \\
\text { sulfamethoxazole }\end{array}$ & $\begin{array}{l}80 \mathrm{mg} / 400 \mathrm{mg} \text { per tablet, } 2 \\
\text { tablets twice daily }\end{array}$ & 5 months \\
\hline Azithromycin & $1 \mathrm{~g}$ weekly & 5 weeks \\
\hline Fluconazole & $150 \mathrm{mg}$ daily & 5 weeks \\
\hline Pregabalin & $\begin{array}{l}\text { Initial dosage of } 75 \mathrm{mg} \text { two } \\
\text { times daily for } 1 \text { week, then } \\
150 \mathrm{mg} \text { two times daily }\end{array}$ & 8 months \\
\hline
\end{tabular}

and integrity of any part of the work have been appropriately investigated and resolved.

Competing interests None declared.

Patient consent Obtained.

Provenance and peer review Not commissioned; externally peer reviewed.

(C) BMJ Publishing Group Ltd (unless otherwise stated in the text of the article) 2018. All rights reserved. No commercial use is permitted unless otherwise expressly granted. 


\section{REFERENCES}

1 Englestein ES, Ruderman MI, Troiano RA, et al. Dilated tonic pupils in neurosyphilis. J Neurol Neurosurg Psychiatry

1986;49:1455-7.
2 Sakai T, Shikishima K, Mizobuchi T, et al. Bilateral tonic pupils associated with neurosyphilis. Jpn J Ophthalmo/ 2003;47:368-71.

3 He D, Jiang B, Binghu J. Syphilitic myelitis: magnetic resonance imaging features. Neurol India 2014;62:89-91.

Copyright 2018 BMJ Publishing Group. All rights reserved. For permission to reuse any of this content visit

http://group.bmj.com/group/rights-licensing/permissions.

BMJ Case Report Fellows may re-use this article for personal use and teaching without any further permission.

Become a Fellow of BMJ Case Reports today and you can:

- Submit as many cases as you like

- Enjoy fast sympathetic peer review and rapid publication of accepted articles

- Access all the published articles

Re-use any of the published material for personal use and teaching without further permission

For information on Institutional Fellowships contact consortiasales@bmjgroup.com

Visit casereports.bmj.com for more articles like this and to become a Fellow 